\title{
DIAGNOSIS OF DISCRETE-TIME SINGULARLY PERTURBED SYSTEMS BASED ON SLOW SUBSYSTEM
}

\author{
Adel TELLILI ${ }^{*+*}$, Nouceyba ABDELKRIM ${ }^{* * *}$, Bahaa JAOUADI", Mohamed Naceur ABDELKRIM"*;** \\ *Unité de Recherche MACS, Ecole Nationale d'Ingénieurs de Gabès, Avenue Omar Ibn El Khattab - Zerig - 6029 Gabès, University of Gabès, Tunisia \\ "Institut Supérieur des Systèmes Industriels de Gabès, Rue, Slaheddine El Ayoubi - 6032 Gabès, University of Gabès, Tunisia \\ ${ }^{* * *}$ Institut Supérieur des Etudes Technologiques de Djerba, Avenue 7 novembre - 4116 Midoun Jerba, Tunisia \\ ${ }^{* * *}$ Ecole Nationale d'Ingénieurs de Gabès, Avenue Omar Ibn El Khattab - Zerig - 6029 Gabès, University of Gabès, Tunisia \\ adel_tellili@lycos.com, nouceyba.naceur@laposte.net, jaouadibahaa@yahoo.com, naceur.abdelkrim@enig.rnu.tn
}

\begin{abstract}
This paper deals with the diagnosis of discrete-time singularly perturbed systems presenting two time scales property. Parity space method is considered to generate the fault detection residual. The focus is in two directions. First, we discuss the residual illconditioning caused by the singular perturbation parameter. Then, the use of the slow subsystem is considered to make the fault diagnosis easier. It is shown that the designed diagnostic algorithm based on reduced order model is close to the one synthesized using the full order system. The developed approach aims at reducing the computational load and the ill-conditioning for stiff residual generation problem. Two examples of application are used to demonstrate the efficiency of the proposed method.
\end{abstract}

Key words: Discrete-Time Singularly Perturbed System, Two Time Scales Property, Model Based Fault Diagnosis, Parity Space Approach, Slow Subsystem

\section{INTRODUCTION}

Singular perturbation systems involving in their realistic representation several small parameters like unmodelled parasitic, capacitances, time and inertia constants, found their applicability in chemical reactors, power system dynamics, control of large scale systems and similar settings (Khalil, 1987; Kokotovic et al., 1986; Tellili et al., 2007). The presence of high dimensionality and ill-conditioning in such systems, gives rise to difficulties in their fault diagnosis and control. These problems are considerably simplified if a decomposition of fast and slow dynamics can be achieved.

Singularly perturbed discrete-time systems are described through three models. The slow and the fast sampling rate models which are obtained through discretization of the singularly perturbed continuous-time system and the pure one which is discrete in nature (Litkouhi and Khalil, 1985; Naidu et al., 1987).

The vulnerability of those time-scale systems, like other automated complex systems, to malfunctions in control actuators, measurement sensors and process equipment requires the design of diagnosis methods to detect and isolate faults. Several approaches have been developed to design diagnosis systems using model-based methods like observer based methods (Frank, 1990; Patton and Chen, 1997), parameter estimation (Clark et al., 1975; Isermann, 1993; Pana and Stoian, 2008) and the parity relation approach which is one of the most commonly used techniques in fault detection and isolation (Chow and Willsky, 1984; Gertler, 1997; Patton and Chen, 1991). Many authors were interested in the fault diagnosis of singularly perturbed systems. Tellili et al. (2004) considered the fast subsystem as a modeling error and generated residuals by using robust parity space in order to detect and isolate faults in singularly perturbed systems. Gong and Khorasani (2005) used the observers relating to the slow and fast reduced subsystems to generate fault diagnosis algorithm in order to detect and isolate actuator faults in continuous singularly perturbed systems. Also Oloomi et al. (2004) employed Chang transformation to separate dynamics of continuous singularly perturbed systems and the original observer based fault diagnosis filter will be approximated by the slow and fast filters. Mease (2005) discusses the use of Lyapunov exponents and vectors to diagnose of the non-linear singularly perturbed systems in flight dynamics.

In this paper, the problem of fault diagnosis of discrete singularly perturbed systems is studied by designing residuals based on reduced slow subsystem. Using parity space approach, the residual for the original system will be generated and then reduced in order to be decoupled from singular perturbation parameter.

\section{SYSTEM DESCRIPTION AND PROBLEM STATEMENT}

Consider the following discrete-time singularly perturbed system (Litkouhi and Khalil, 1985; Naidu et al., 1987; Kafri and Abed, 1996):

$\left\{\begin{array}{l}{\left[\begin{array}{l}\mathrm{x}_{1}(k+1) \\ \mathrm{x}_{2}(k+1)\end{array}\right]=\left[\begin{array}{ll}\mathrm{A}_{11} & \varepsilon \mathrm{A}_{12} \\ \mathrm{~A}_{21} & \varepsilon \mathrm{A}_{22}\end{array}\right]\left[\begin{array}{l}\mathrm{x}_{1}(k) \\ \mathrm{x}_{2}(k)\end{array}\right]+\left[\begin{array}{l}\mathrm{B}_{1} \\ \mathrm{~B}_{2}\end{array}\right] \mathrm{u}(k)} \\ \mathrm{y}(k)=\left[\begin{array}{ll}\mathrm{C}_{1} & \varepsilon C_{2}\end{array}\right]\left[\begin{array}{l}\mathrm{x}_{1}(k) \\ \mathrm{x}_{2}(k)\end{array}\right]\end{array}\right.$

where: $\mathrm{x}_{1}(k) \in R^{n_{1}}, \mathrm{x}_{2}(k) \in R^{n_{2}}$, are state vectors, $\mathrm{y}(k) \in$ $R^{p}$ is the output, and $\mathrm{u}(k) \in R^{m}$ is the control input. The singular perturbation parameter $\varepsilon$ satisfies $0<\varepsilon<1$. All matrices are assumed to have appropriate dimensions.

The above description is obtained by setting $i$ and $j$ to zero in the following model: 


$$
\left\{\begin{array}{c}
{\left[\begin{array}{l}
\mathrm{x}_{1}(k+1) \\
\varepsilon^{2 i} \mathrm{x}_{2}(k+1)
\end{array}\right]=\left[\begin{array}{ll}
\mathrm{A}_{11} & \varepsilon^{1-j} \mathrm{~A}_{12} \\
\varepsilon^{j} \mathrm{~A}_{21} & \varepsilon \mathrm{A}_{22}
\end{array}\right]\left[\begin{array}{l}
\mathrm{x}_{1}(k) \\
\mathrm{x}_{2}(k)
\end{array}\right]+} \\
{\left[\begin{array}{l}
\mathrm{B}_{1} \\
\varepsilon^{j} \mathrm{~B}_{2}
\end{array}\right] \mathrm{u}(k)} \\
\mathrm{y}(k)=\left[\begin{array}{ll}
\mathrm{C}_{1} & \varepsilon^{1-j} \mathrm{C}_{2}
\end{array}\right]\left[\begin{array}{l}
\mathrm{x}_{1}(k) \\
\mathrm{x}_{2}(k)
\end{array}\right]
\end{array}\right.
$$

which represents a pure singularly perturbed discrete system issued from the difference equations introducing a small positive parameter $\varepsilon$ in the state vector (Naidu et al., 1987). Corresponding slow and fast subsystems can be obtained from the original system by singular perturbation approach or by block diagonalization (Litkouhi and Khalil, 1985; Naidu et al., 1987). The two approaches give identical results.

To derive the slow subsystem, it is assumed that the fast variables have reached their established regime by setting $\varepsilon=0$. Then, we get the slow subsystem of dimension $n_{1}$ as:

$$
\left\{\begin{array}{l}
\mathrm{x}_{l}(k+1)=\mathrm{A}_{11} \mathrm{x}_{l}(k)+\mathrm{B}_{1} \mathrm{u}_{l}(k) \\
\mathrm{y}_{l}(k)=\mathrm{C}_{1} \mathrm{x}_{l}(k) \\
\mathrm{x}_{l}\left(k_{0}\right)=\mathrm{x}_{10}
\end{array}\right.
$$

The fast subsystem of dimension $n_{2}$, obtained by considering that the slow variables are constant during the fast transients, is given by:

$$
\left\{\begin{array}{l}
\mathrm{x}_{r}(k+1)=\mathrm{A}_{r} \mathrm{x}_{r}(k)+\mathrm{B}_{r} \mathrm{u}_{r}(k) \\
\mathrm{y}_{r}(k)=\mathrm{C}_{r} \mathrm{x}_{r}(k)+\mathrm{D}_{r} \mathrm{u}_{r}(k) \\
\mathrm{x}_{r}\left(k_{0}\right)=\mathrm{x}_{20}-\mathrm{A}_{22}^{-1} \mathrm{~A}_{21} \mathrm{x}_{10}
\end{array}\right.
$$

where: $\mathrm{A}_{r}=\varepsilon\left(\mathrm{A}_{22}-\mathrm{A}_{21} \mathrm{~A}_{11}^{-1} \mathrm{~A}_{12}\right), \mathrm{B}_{r}=\mathrm{B}_{2}-\mathrm{A}_{21} \mathrm{~A}_{11}^{-1} \mathrm{~B}_{1}$, $\mathrm{C}_{r}=\varepsilon\left(\mathrm{C}_{2}-C_{1} \mathrm{~A}_{11}^{-1} \mathrm{~A}_{12}\right), \mathrm{D}_{r}=-\mathrm{C}_{1} \mathrm{~A}_{11}^{-1} \mathrm{~B}_{1}, \mathrm{u}_{l}$ and $\mathrm{u}_{r}$ verify $\mathrm{u}=\mathrm{u}_{l}+\mathrm{u}_{r}$ and represent respectively the fast and slow components of the input $u$.

Denote:

$$
\begin{aligned}
& \mathrm{A}(\epsilon)=\left[\begin{array}{ll}
\mathrm{A}_{11} & \varepsilon \mathrm{A}_{12} \\
\mathrm{~A}_{21} & \varepsilon \mathrm{A}_{22}
\end{array}\right], \mathrm{B}=\left[\begin{array}{l}
\mathrm{B}_{1} \\
\mathrm{~B}_{2}
\end{array}\right], \mathrm{C}(\varepsilon)=\left[\begin{array}{ll}
\mathrm{C}_{1} & \varepsilon \mathrm{C}_{2}
\end{array}\right] \\
& \text { and } \mathrm{x}(k)=\left[\begin{array}{l}
\mathrm{x}_{1}(k) \\
\mathrm{x}_{2}(k)
\end{array}\right] .
\end{aligned}
$$

Then system (1) can be rewritten, in presence of additive fault $\boldsymbol{f}(k)$, as follows:

$\left\{\begin{array}{l}\dot{\mathrm{x}}(k)=\mathrm{A}(\varepsilon) \mathrm{x}(k)+\mathrm{B} \mathrm{u}(k)+\mathrm{E}_{1} \mathrm{f}(k) \\ \mathrm{y}(k)=\mathrm{C}(\varepsilon) \mathrm{x}(k)+\mathrm{E}_{2} \mathrm{f}(k)\end{array}\right.$

where $E_{2}$ and $E_{2}$ are known as fault entry matrices of appropriate dimensions.

The problem under consideration is to design a fault diagnosis algorithm through the construction of an appropriate residual based on parity space method.

\section{RESIDUAL GENERATION BASED ON THE ORIGINAL SYSTEM}

The parity equations are obtained by calculating the outputs of the singularly perturbed systems (5) over an horizon $s \in \mathbb{N}^{*}$, that leads to the following relationship (Chow and Willsky, 1984):

$$
\begin{gathered}
\mathrm{Y}(k, s)=\mathrm{H}(s, \varepsilon) \mathrm{x}(k-s)-\mathrm{G}(s, \varepsilon) \mathrm{U}(k, s)+ \\
\mathrm{E}(s, \varepsilon) \mathrm{F}(k, s)
\end{gathered}
$$

$$
\begin{aligned}
& \text { where: } \mathrm{F}(k, s)=\left[\begin{array}{lllll}
\mathrm{f}^{T}(k-s) & \mathrm{f}^{T}(k-s+1) & \ldots & \mathrm{f}^{T}(k)
\end{array}\right]^{T} \text {, } \\
& \mathrm{Y}(k, s)=\left[\begin{array}{lllll}
\mathrm{y}^{T}(k-s) & \mathrm{y}^{T}(k-s+1) & \ldots & \mathrm{y}^{T}(k)
\end{array}\right]^{T}, \\
& \mathrm{U}(k, s)=\left[\begin{array}{lllll}
\mathrm{u}^{T}(k-s) & \mathrm{u}^{T}(k-s+1) & \ldots & \mathrm{u}^{T}(k)
\end{array}\right]^{T}, \\
& \mathrm{G}(s, \varepsilon)= \\
& {\left[\begin{array}{lllll}
0 & 0 & \cdots & \cdots & 0 \\
\mathrm{C}(\varepsilon) \mathrm{B} & 0 & 0 & \cdots & 0 \\
\mathrm{C}(\varepsilon) \mathrm{A}(\varepsilon) \mathrm{B} & \mathrm{C}(\varepsilon) \mathrm{B} & 0 & \cdots & 0 \\
\vdots & \vdots & \vdots & \vdots & \vdots \\
\mathrm{C}(\varepsilon) \mathrm{A}^{s-1}(\varepsilon) \mathrm{B} & \mathrm{C}(\varepsilon) \mathrm{A}^{s-2}(\varepsilon) \mathrm{B} & \cdots & \mathrm{C}(\varepsilon) \mathrm{B} & 0
\end{array}\right]} \\
& \mathrm{H}(s, \varepsilon)=\left[\begin{array}{l}
\mathrm{C}(\varepsilon) \\
\mathrm{C}(\varepsilon) \mathrm{A}(\varepsilon) \\
\vdots \\
\mathrm{C}(\varepsilon) \mathrm{A}^{s}(\varepsilon)
\end{array}\right] \text { and } \\
& \mathrm{E}(s, \varepsilon)= \\
& {\left[\begin{array}{lllll}
\mathrm{E}_{2} & 0 & \cdots & \cdots & 0 \\
\mathrm{C}(\varepsilon) \mathrm{E}_{1} & \mathrm{E}_{2} & 0 & \cdots & 0 \\
\mathrm{C}(\varepsilon) \mathrm{A}(\varepsilon) \mathrm{E}_{1} & \mathrm{C}(\varepsilon) \mathrm{E}_{1} & \mathrm{E}_{2} & \cdots & 0 \\
\vdots & \vdots & \vdots & \vdots & \vdots \\
\mathrm{C}(\varepsilon) \mathrm{A}^{s-1}(\varepsilon) \mathrm{E}_{1} & \mathrm{C}(\varepsilon) \mathrm{A}^{s-2}(\varepsilon) \mathrm{E}_{1} & \cdots & \mathrm{C}(\varepsilon) \mathrm{E}_{1} & \mathrm{E}_{2}
\end{array}\right]}
\end{aligned}
$$

The choice of the horizon $\mathrm{s}$ must provide a maximum degree of freedom in designing fault isolation scheme and have to improve its performance (Chow and Willsky, 1984; Li and Shah, 2002). In deterministic case, it is sufficient to set $s=n$ where $n$ is the state dimension, to ensure efficient fault detection algorithm (Maquin and Ragot, 2000).

A parity relation based residual generator can be designed as:

$\mathrm{R}(k, \varepsilon)=\Omega(\varepsilon)(\mathrm{Y}(k, s)-\mathrm{G}(s, \varepsilon) \mathrm{U}(k, s))$

The dynamics of residual generator (5) are governed by:

$\mathrm{R}(k, \varepsilon)=\Omega(\varepsilon) \mathrm{E}(s, \varepsilon) \mathrm{F}(k, \varepsilon)$

where: $\mathrm{R}(k, s)$ is the residual signal, $\Omega(k, s)$ is the parity vector which satisfies:

$$
\Omega(\varepsilon) \mathrm{H}(s, \varepsilon)=0 \text { and } \Omega(\varepsilon) \mathrm{E}(s, \varepsilon) \neq 0
$$

Under the assumption that the system parameters, in absence of fault, do not change, the residual $\mathrm{R}(k, s)$ verifies the following properties:

- $\mathrm{R}(k, s)=0$ in fault free case;

- $\mathrm{R}(k, s) \neq 0$ in presence of fault.

The so generated residual must be independent of the singular perturbation parameter $\varepsilon$ to avoid the ill-conditioning and numerical difficulties. To alleviate this ill-conditioning and to reduce the amount of computation, reduction of the residual will be considered.

\section{RESIDUAL REDUCTION}

The reduction of the full order residual (5) is done by setting the singular perturbation parameter to zero. Eliminating $\varepsilon$ from the matrices $\mathrm{G}(s, \varepsilon), \mathrm{H}(s, \varepsilon)$ and $\mathrm{E}(s, \varepsilon)$, we get:

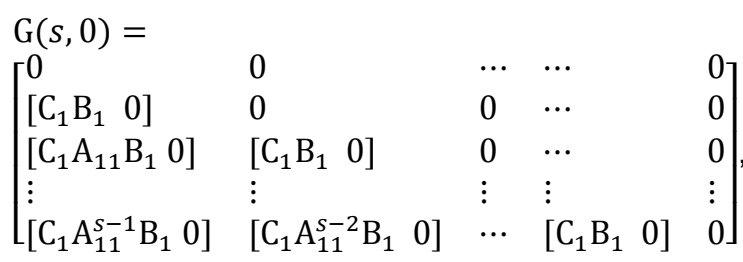




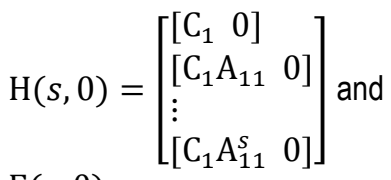

$$
\begin{aligned}
& \mathrm{E}(s, 0)=
\end{aligned}
$$

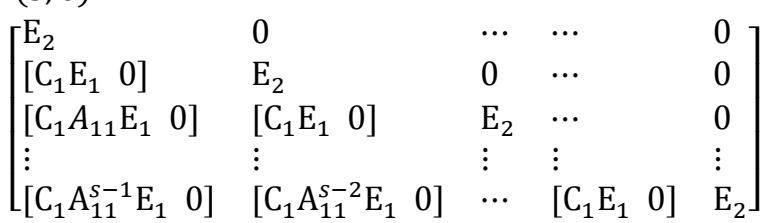

The above calculated residual matrices are $\varepsilon$ independent, they correspond to the matrices determined using the slow subsystem (3): $\mathrm{H}(k, 0)=\mathrm{H}_{s}(k), \mathrm{G}(k, 0)=\mathrm{G}_{s}(k)$ and $\mathrm{E}(k, 0)=$ $\mathrm{E}_{s}(k)$. Consequently, we get the reduced residual $\mathrm{R}(k, 0)$ which matches the slow subsystem based residual $\mathrm{R}_{s}(k): \mathrm{R}(k, 0)=$ $\mathrm{R}_{s}(k)$. It follows that additive faults diagnosis in discrete-time singularly perturbed system can be achieved using residual generated based on slow subsystem. That leads to the following proposition:

Proposition: Consider the discrete-time singularly perturbed system described by equation (1), the slow subsystem related to the original system is represented by equation (3). Under the assumption that the additive faults attack the system in steady state, additive fault detection and isolation of the original system can be ensured by residual generated based on slow subsystem and using parity space approach. It can be expressed as follows:

$\mathrm{R}_{s}(k)=\Omega_{s}\left(\mathrm{Y}_{s}(k)-\mathrm{G}_{s}(s) \mathrm{U}_{s}(k)\right)$

where all matrices are calculated based on the slow subsystem.

Consequently, the residual is independent of the singular perturbation parameter $\varepsilon$ and generated using the slow subsystem model which order is less than the one of the original singularly perturbed system. By analogy with the commutativity of decomposition and discretization (Kenneth and David, 1992), we have shown that residual generation followed by reduction and system reduction followed by residual generation are commutative operations. This is shown in the following scheme:

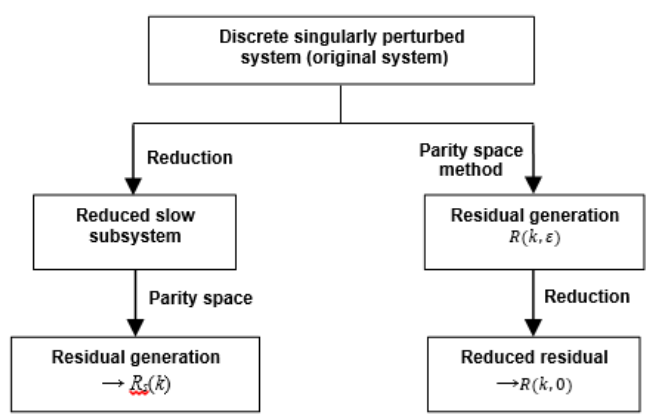

Fig. 1. Commutativity of residual generation and reduction

\section{EXAMPLES OF APPLICATION}

The following two examples illustrate the effectiveness of the derived diagnosis approach.

\subsection{Example 1}

The first example is based on a real laboratory two tanks system. We consider the following linear time-invariant discrete-time singularly perturbed system modeling the two tanks system with single input and single output:

$$
\begin{aligned}
& \left(\left[\begin{array}{l}
\tilde{\mathrm{x}}_{1}(k+1) \\
\tilde{\mathrm{x}}_{2}(k+1)
\end{array}\right]=\left[\begin{array}{rr}
0.9991 & 0.0014 \varepsilon \\
0.0705 & 7.7766 \varepsilon
\end{array}\right]\left[\begin{array}{l}
\tilde{\mathrm{x}}_{1}(k) \\
\tilde{\mathrm{x}}_{2}(k)
\end{array}\right]+\right. \\
& \left\{\begin{array}{r}
{\left[\begin{array}{l}
49.7359 \\
3895.1
\end{array}\right] \mathrm{u}(k)} \\
\mathrm{y}(k)=\left[\begin{array}{ll}
1 & 0
\end{array}\right]\left[\begin{array}{l}
\tilde{\mathrm{x}}_{1}(k) \\
\tilde{\mathrm{x}}_{2}(k)
\end{array}\right]
\end{array}\right.
\end{aligned}
$$

The discrete slow subsystem is obtained as:

$$
\left\{\begin{array}{l}
\tilde{\mathrm{x}}_{l}(k+1)=0.9991 \tilde{\mathrm{x}}_{l}(k)+49.7359 \mathrm{u}_{l}(k) \\
\mathrm{y}_{l}(k)=\tilde{\mathrm{x}}_{l}(k) \\
\tilde{\mathrm{x}}_{l}(0)=0
\end{array}\right.
$$

The residual $R_{s}(k)$ is generated based on the slow subsystem and using the parity space method. It is governed by:

$$
\begin{aligned}
R_{s}(k)=-5.437 * 10^{-4} y(k-1)+ \\
5.441 * 10^{-4} y(k)+0.0271 u(k)
\end{aligned}
$$

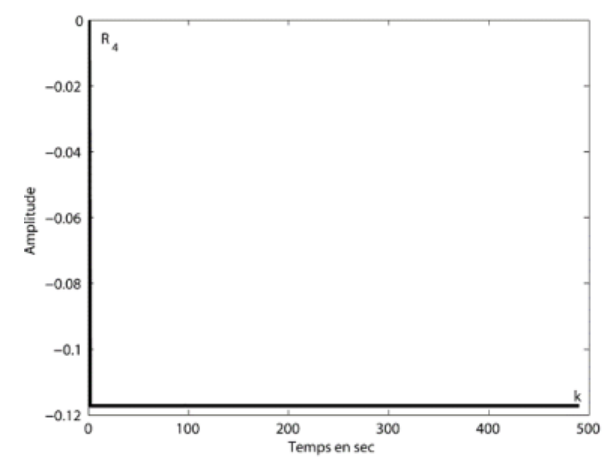

Fig. 2. Evolution of the residual $R_{S}(k)$ in fault free case

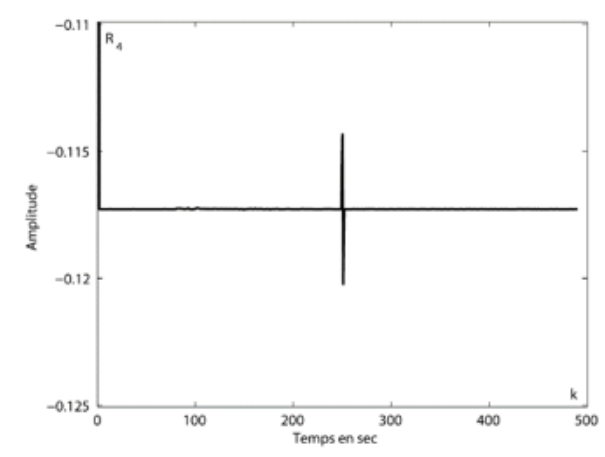

Fig. 3. Residual $R_{S}(k)$ after fault occurring

The Fig. 2 represents the residual time evolution in the fault free case. It shows that the residual do not presents remarkable deviations. But they take values different from zero, which can be explained by modelling and reduction errors.

The generation of an abrupt sensor fault at time instant 250 seconds leads to the time evolution of the residual $R_{s}(k)$ illustrated by Fig. 3. It shows a deviation at time $250 \mathrm{sec}$, which indicates the presence of fault. This result demonstrates that the residual generated based on the slow subsystem is sensitive to sensor additive fault affecting the original singularly perturbed system. This result makes the fault diagnosis of high order systems easier. 
The real application is based on a real laboratory application. It is composed of three identical water tanks (see Fig. 4). A detailed model of this application has been presented in (Abdelkrim and Tellili, 2009). Only two tanks are considered.

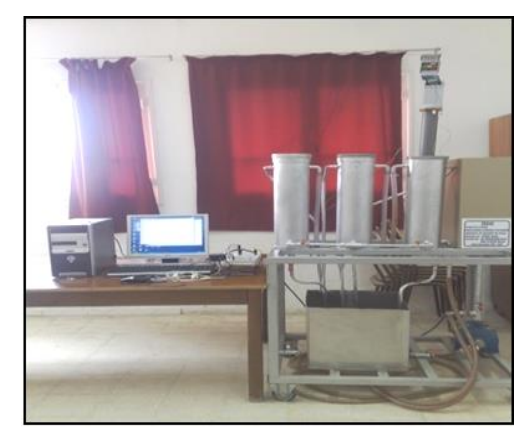

Fig. 4. Three tanks hydraulic system

The external input flow is considered as control input and The water levels are represented by the states $x_{1}(t)$ and $x_{2}(t)$. Using the parity space method, the residual trajectory in the fault free case is shown in Fig. 5.

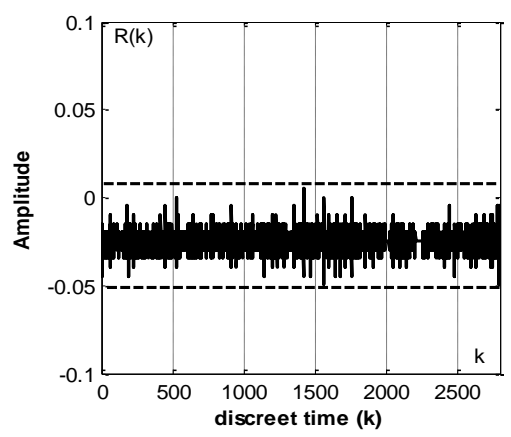

Fig. 5. Real time residual in fault free case

The residual shown in Fig. 5 takes values different from zero in spite of the absence of faults. This is due to several factors as the modelling errors and the disturbances affecting the real system. A threshold will be fixed over the fluctuations to avoid false alarms. A sensor fault is generated at time instance $k=2500$ (which corresponds to $t=250 \mathrm{sec}$ ). The resulting residual behaviour is shown in Fig. 6 .

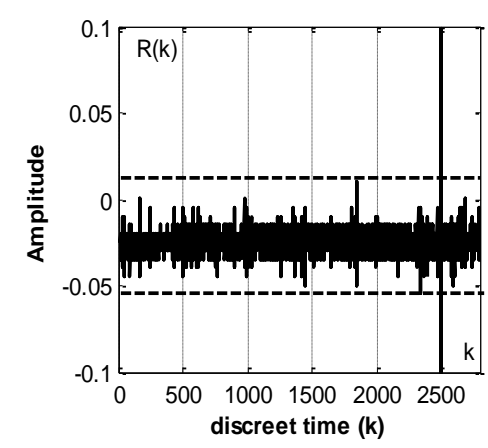

Fig. 6. Real time residual in presence of fault

It is clear on Figs. 5 and 6 that the presence of the fault at time $k=2500$ induces a residual deviation which exceeds the fixed threshold. These results show that the residual generated on the basis of the discrete-time model, is able to detect the faults affecting the real system.

\subsection{Example 2}

In this second example, a discrete-time singularly perturbed system with two outputs and one input is considered. It is described by equation (1) with:

$$
\begin{aligned}
& \mathbf{A}_{11}=\left[\begin{array}{cc}
-0.2 & 0.1 \\
0.1 & -0.2
\end{array}\right], \mathbf{A}_{12}=\left[\begin{array}{cc}
-1 & 0.2 \\
-1 & 0.4
\end{array}\right], \mathbf{B}_{1}=\left[\begin{array}{c}
-1 \\
4
\end{array}\right], \\
& \mathbf{A}_{21}=\left[\begin{array}{cc}
0.6 & -0.5 \\
0.05 & -0.2
\end{array}\right], \mathbf{A}_{22}=\left[\begin{array}{cc}
-0.1 & 0.03 \\
0.1 & -0.1
\end{array}\right], \mathbf{B}_{2}=\left[\begin{array}{l}
1 \\
2
\end{array}\right], \\
& \mathbf{C}_{1}=\left[\begin{array}{cc}
-1 & 2 \\
0 & 2
\end{array}\right] \text { and } \mathbf{C}_{2}=\left[\begin{array}{cc}
-0.1 & 3 \\
1 & 0.2
\end{array}\right] .
\end{aligned}
$$

The first simulation depicts how close is the global system output to the slow subsystem output for various values of the singular perturbation parameter $\varepsilon$.

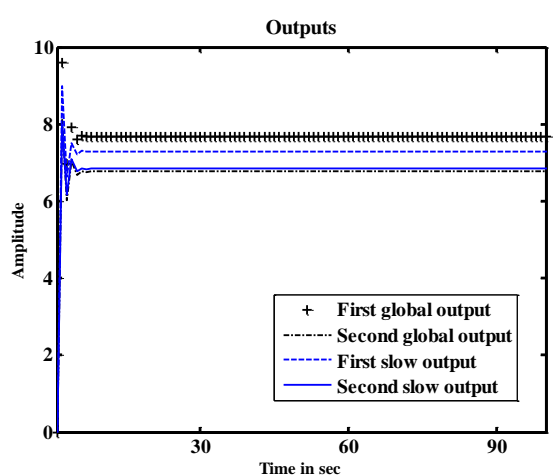

Fig. 7. Global system und slow subsystem outputs by $\varepsilon=0.1$

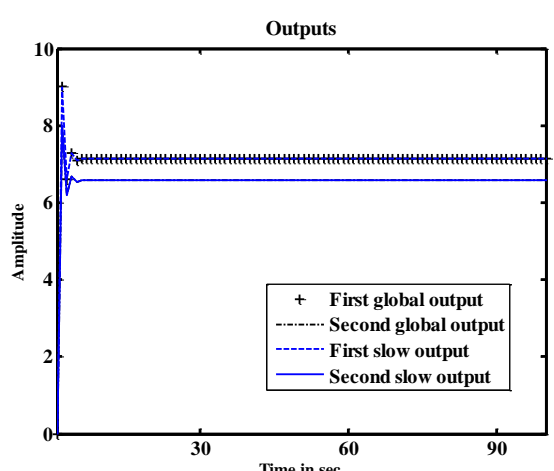

Fig. 8. Global system und slow subsystem outputs by $\varepsilon=0.001$

It is clear in Figs. 7 and 8 that the approximation of the global system with the slow subsystem is better if the singular perturbation parameter $\varepsilon$ is smaller. This simulation result is obvious because the slow subsystem is obtained by letting $\varepsilon$ to zero. The residual will be then generated using the slow subsystem obtained by applying the singular perturbation method. Knowing that for sufficient small singular perturbation parameter the slow subsystem provides an approximation of the global system, the residual is designed using the slow subsystem matrices and makes use of the global system input and outputs leading to the following residual vector: 
$\mathrm{R}(k)=\left[\begin{array}{c}\mathrm{R}_{l 1}(k)=0.3589 \mathrm{y}_{1}(k)-0.1368 \mathrm{y}_{2}(k)+0.9256 \mathrm{y}_{1}(k+1)-0.0337 \mathrm{y}_{2}(k+1)+0.026 \mathrm{y}_{1}(k+2) \\ \quad+0.0155 \mathrm{y}_{2}(k+2)-7.9704 \mathrm{u}(k)-0.3578 \mathrm{u}(k+1) \\ \mathrm{R}_{l 2}(k)=-0.0718 \mathrm{y}_{1}(k)+0.0274 \mathrm{y}_{2}(k)+0.0155 \mathrm{y}_{1}(k+1)+0.0067 \mathrm{y}_{2}(k+1)-0.0052 \mathrm{y}_{1}(k+2) \\ \quad+\mathrm{y}_{2}(k+2)+1.5941 \mathrm{u}(k)-7.9533 \mathrm{u}(k+1)\end{array}\right]$

The theoretical signature fault matrix, which reflects the residual sensitivity against faults, will be used later to locate the fault (fault isolation). It is given by:

$$
\Sigma=\left[\begin{array}{llll}
y_{1} & y_{2} & u \\
-1 & -1 & -1 \\
-1 & 1 & -1
\end{array}\right] \quad R_{l 1}
$$

The above mentioned incidence matrix can be interpreted as follow: a positive deviation of the first residual and a negative deviation of the second residual indicates the presence of fault in the first sensor $\left(y_{1}\right)$ and so forth. In the fault free case, the residuals must be close to zero. In fact, Fig. 9 shows the time evolution of the slow subsystem based residuals in absence of failures, there are no deviations.
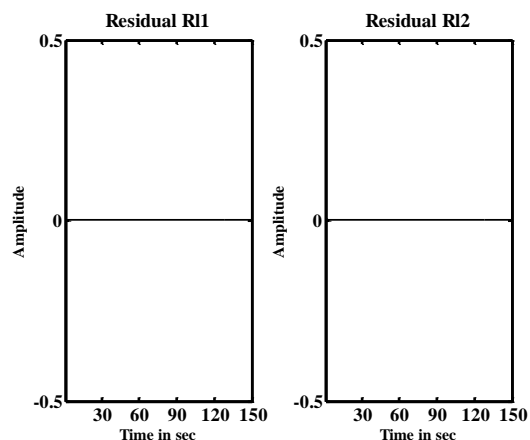

Fig. 9. Residuals in fault free case by $\varepsilon=0.001$
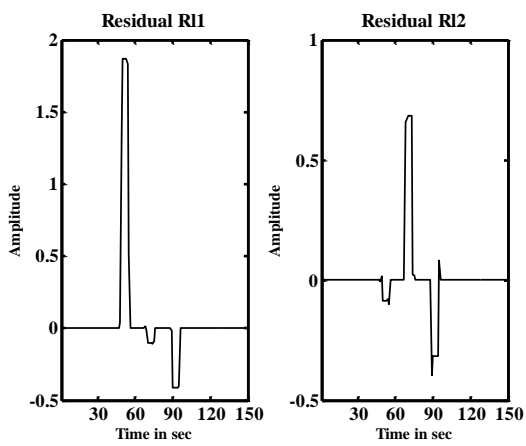

Fig. 10. Residuals in faulty case by $\varepsilon=0.001$

Three additive faults, modelled by a measurement bias, occur in sensor $\left(\mathrm{y}_{1}\right)$ from $t=50 \mathrm{sec}$ to $t=55 \mathrm{sec}$, in sensor $\left(\mathrm{y}_{2}\right)$ between $t=70 \mathrm{sec}$ and $t=75 \mathrm{sec}$ and finally in actuator (u) from $t=90 \mathrm{sec}$ sec to $t=95 \mathrm{sec}$. Fig. 10 shows the time evolution of the residuals after fault happening. The values of both residuals at the considered time intervals underline the abnormal behaviour of the global system which indicates the residuals sensitivity towards the considered additive faults.

Once the faults are detected in the plant, the next step is to locate them through the instantaneous fault signatures which reflect the residuals behaviour after fault occurrence and take in this case the following values: $S_{50-55}=\left[\begin{array}{l}1 \\ -1\end{array}\right], S_{70-75}=$ $\left[\begin{array}{l}-1 \\ 1\end{array}\right]$ and $S_{90-95}=\left[\begin{array}{l}-1 \\ -1\end{array}\right]$. The comparison between theoretical and instantaneous fault signatures allows accurate fault isolation.

\subsection{Interpretation and discussion}

The above examples illustrate the developed method for the fault diagnosis of discrete-time singularly perturbed system based on the slow reduced subsystem. The designed algorithm is independent of the singular perturbation parameter $\varepsilon$ (equation 14). The developed residuals show deviations further to the occurrence of additive sensor and actuator faults (Fig.10) which allows their detection and isolation.
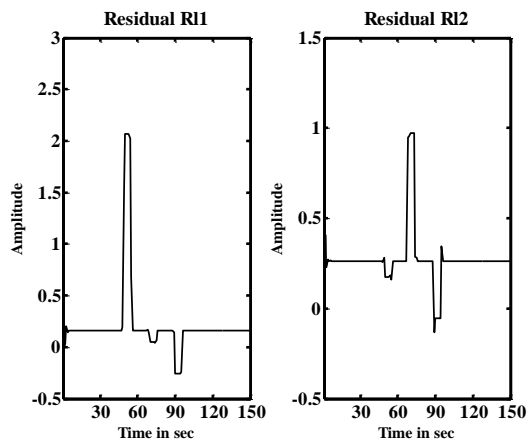

Fig. 11. Residuals in faulty case by $\varepsilon=0.1$
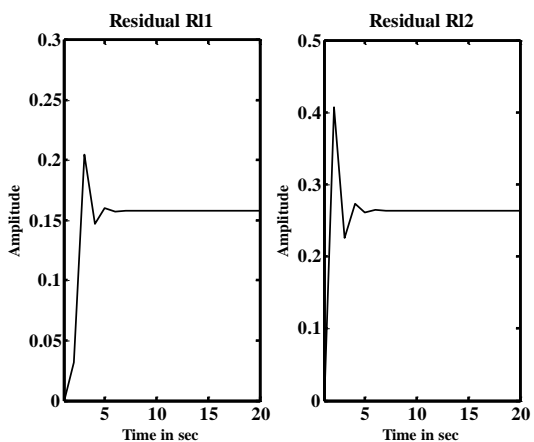

Fig. 12. Residuals in fault free case by $\varepsilon=0.1$
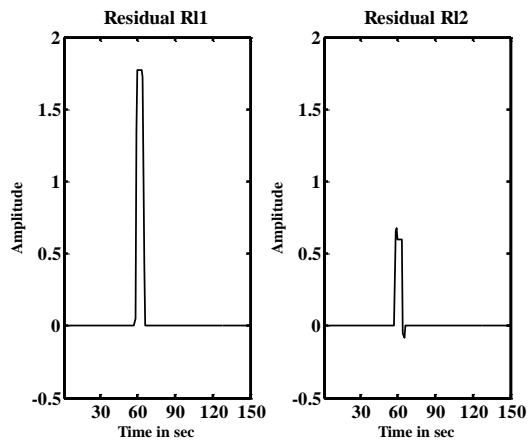

Fig. 13. Residuals in presence of simultaneous sensors faults 
However, there are some constraints to respect. First, the singular perturbation parameter must be enough small to guaranty a gut approximation of the global system through the slow subsystem (Figs. 7 and 8); otherwise the residuals cannot be close to zero in absence of faults (risk of false alarm). In this case the residuals remain still sensitive to faults (Fig. 11). The second point concerns the residuals behaviour at the beginning ( 5 first seconds), they take values different from zero in spite of fault absence, which can lead to false alarms (Fig.12). So during the five first seconds in the second example, it is assumed that no faults happen. Finally the main disadvantage of the used parity space approach is that only failures occurring in different time intervals can be isolated. For example, if additive failures affect simultaneously the first and the second sensor between $t=50 \mathrm{sec}$ and $t=55 \mathrm{sec}$, the residuals detect the presence of fault during this period but the location of the faulty element using the theoretical and the instantaneous fault signatures is no longer possible (Fig.13).

\section{CONCLUSION}

In this paper we study model based fault diagnosis of discretetime singularly perturbed system. The residual is generated using parity space method and depends on the singular perturbation parameter. The ill-conditioning problem of the residual fault generator is solved by the residual synthesis using the slow subsystem. In fact, the reduced order subsystem is independent from singular perturbation parameter and provides an approximation of the original system. A two water tanks application example has shown that the residual generated based on the slow subsystem is able to detect sensor fault occurred in the original singularly perturbed system. A second example is carried out to show the ability of the method to deal with multiple faults.

\section{REFERENCES}

1. Abdelkrim N., Tellili A. (2009), Model based fault diagnosis of a hydraulic system using singular perturbation approach, International Review of Automatic Control (I.RE.A.CO), Vol. 2, No. 6, 731-737.

2. Chow E., Willsky A. (1984), Analytical redundancy and the design of robust failure detection systems, IEEE Transactions on Automatic Control, Vol. 29, No. 7, 603-614.

3. Clark R. N., Fasth D. C., Walton W. M. (1975), Detecting instrument malfunctions in control systems, IEEE Transactions on Automatic Control, Vol. AES-16, No. 4, 468-473.

4. Frank P. M. (1990), Fault diagnosis in dynamic systems using analytical and knowledge-based redundancy, A survey and some new results, Automatica, Vol. 26, No. 3, 459-474.

5. Gertler J. (1997), Fault detection and isolation using parity relations, Control Engineering Practice, Vol. 5, No. 5, 653-661.

6. Gong F., Khorasani K. (2005), Fault Diagnosis of Linear Singularly Perturbed Systems, Proceedings of the 44th IEEE Conference on Decision and Control, and the European Control Conference, Seville, Spain.

7. Isermann R. (1993), Fault diagnosis of machines via parameter estimation and knowledge processing, Automatica, Vol. 29, No.4, 815-835.

8. Kafri W., Abed E. (1996), Stability analysis of discrete-time singularly perturbed systems, IEEE Trans. On Circuits and Systems, Vol. 43, No.10, $848-850$.
9. Kenneth R. S., David G. T. (1992), On the commutativity of decomposition and discretization for linear singularly perturbed systems, IEEE Proceedings of the $31^{\text {st }}$ Conference on decision and control, Arizona.

10. Khalil H. K. (1987), Output feedback control of linear two-time-scale systems, IEEE Transactions on Automatic Control, Vol. AC-32, No. 9, 784-792.

11. Kokotovic P., Khalil H., O'Reilly J. (1986), Singular perturbation methods in control: analysis and design, Academic press, Harcourt Brace Jovanovich Publishers.

12. Li W., Shah S. (2002), Structured residual vector-based approach to sensor fault detection and isolation, Journal of Process Control, Vol. 12, 429-443.

13. Litkouhi B., Khalil H. (1985), Multirate and Composite Control of Two-Time-Scale Discrete-Time Systems, IEEE Transactions on Automatic Control, Vol. 30, No. 7, 645-651.

14. Maquin D., Ragot J. (2000), Diagnostic des systèmes linaires. Hermes-science ed., France.

15. Mease K. D. (2005), Multiple time-scales in nonlinear flight mechanics: diagnosis and modelling, Applied Mathematics and Computation, Vol. 164, No. 2, 627-648.

16. Naidu D. S., Price D. B., Hibey J. L. (1987), Singular perturbations and time scales (SPaTS) in discrete control systems-an overview". Proceedings of the 26th Conference on Decision and Control, Los Angeles, USA.

17. Oloomi H., Saif M., Shafai B. (2004), On the Well Posedness of Singularly Perturbed Fault Detection Filters, Proceeding of the 2004 American Control Conference Boston, Massachusetts.

18. Pana C., Stoian V., (2008), A fault-tolerant control system for a hexapod mobile robot, Acta Mechanica et Automatica, Vol. 2, No. 3, 80-85.

19. Patton R. J., Chen J. (1991), A Review of Parity Space Approaches to Fault Diagnosis, Proceedings of IFAC Symposium on Safeprocess, Vol. 1, 239-255.

20. Patton R. J., Chen J. (1997), Observer-based fault detection and isolation : Robustness and application, Control Engineering Practice, Vol. 5, No. 5, 671-682.

21. Tellili A., Abdelkrim M., Benrjeb M. (2004), Model-based fault diagnosis of two-time scales singularly perturbed systems, IEEE Control, Communications and Signal Processing, 819-822.

22. Tellili A., Abdelkrim M.N., Benrejeb M. (2007), Reliable $\mathrm{H}_{\infty}$ control of multiple time scales singularly perturbed systems with sensor failure, International Journal of Control, Vol. 80, No. 5, 659-665. 